\title{
LAS REDES DE PRÁCTICA COMO ESPACIOS EDUCATIVOS INTEGRALES: LA RED TRAMA3
}

\author{
Bianca Vienni \\ David Barreiro \\ Camila Gianotti ${ }^{(*)}$
}

La red TRAMA3 (Trabajo en Red para la Acción Multivocal en Arqueología, Antropología y Ambiente), financiada por el área de Ciencia y Sociedad del programa CYTED para el período 2013-2016, busca contribuir a la generación de una respuesta a los retos que se le plantean a la arqueología y la antropología, pero también a otras disciplinas (como las ambientales) que tienen al patrimonio como eje y campo transdisciplinario de acción e investigación. La red se concibe como un espacio común para cotejar, intercambiar y ampliar experiencias, una red orientada tanto a la teoría, reflexionando sobre las condiciones de producción y emergencia de los procesos de patrimonialización y su dialéctica como fenómeno social, como a la práctica, fomentando las comunidades de aprendizaje-enseñanza y la generación de valores y actitudes positivas y de transformación en torno al patrimonio, tanto natural como cultural (BARREIRO Y GIANOTTI, 2014).

TRAMA3 está formada por ocho socios científicos de diferentes países así como por una comunidad de usuarios. Cada uno de los socios aporta su experiencia y su contribución en torno a un espacio concreto en el que se desarrolla su actividad científica que denominamos sitios laboratorio. La red está coordinada desde el Laboratorio de Arqueología del Paisaje y Patrimonio (LAPPU), dirigido por Camila Gianotti y alojado en la Facultad de Humanidades y Ciencias de la Educación con una unidad asociada en el Centro Universitario de la Región Este (Universidad de la República), de Uruguay.

Entre los objetivos de la Red TRAMA3 se plantea integrar la discusión/reflexión sobre experiencias y valores asociados al patrimonio dentro del ámbito educativo, formativo y de gestión,

\footnotetext{
${ }^{(*)}$ Bianca Vienni. Doutora em Gestión y Conservación del Patrimonio, Universidad de Granada. Investigadora na Universidad de la República, Uruguay. E-mail: bianca.vienni@lappu.edu.uy.

David Barreiro. Doutor em Arqueologia e Sociedade. Técnico no Instituto de Ciencias del Patrimonio (Incipit), integrante do Consejo Superior de Investigación Científica, Vigo, Espanha.E-mail: david.barreiro@incipit.csic.es.

Camila Gianotti. Doutora em Arqueoloxía, Historia da Antigüidade e Ciencias e Técnicas Historiográficas pela Universidad de Santiago de Compostela. Docente e investigadora na Universidad de la República, Uruguay. E-mail: camila.gianotti@lappu.edu.uy.
} 
fomentando y facilitando la colaboración y enseñanza-aprendizaje entre investigadores, comunidad y gestores del patrimonio en el terreno de la educación formal, no formal e informal.

Bajo este objetivo es que consideramos a la red como un ámbito educativo integral desde dos niveles. Un primer nivel vinculado a las sinergias generadas entre los socios y los equipos de trabajo, donde se construye dialógicamente una red de práctica entendida como aquella que consolida saberes y conocimientos de diversos tipos, no solamente académicos (BROWN Y DUGUID, 2000; BAMMER, 2013). En este nivel, los integrantes directos de la red co-construyen conocimiento desde prácticas integrales (AROCENA, 2008) y multivocales. En un segundo nivel de práctica educativa, TRAMA3 fundamenta su actividad a través de varias dinámicas, a fin de generar sinergias que revaloricen el trabajo de toda la red. Tanto su principal actividad, los seminarios de trabajo, de periodicidad anual, como la realización de dos tipos de productos específicos (los protocolos y las aplicaciones) responden al objetivo sustantivo de co-crear dispositivos educativos para y con el patrimonio.

El presente artículo detalla las acciones llevadas a cabo en los dos niveles mencionados y analiza los resultados obtenidos hasta el momento en el entendido que este proceso educativo se formaliza considerando al patrimonio como un campo transdisciplinario. Se inicia presentando la red y sus socios así como las principales actividades que ha llevado a cabo. Seguidamente, se ejemplifican los dos niveles de este ámbito educativo integral. Para finalizar, presentamos algunas líneas que pueden considerarse como los resultados de ese espacio educativo y que ponderan el valor intrínseco del patrimonio como campo transdisciplinario de acción e investigación.

\section{LA RED TRAMA3}

La red TRAMA3 (Trabajo en Red para la Acción Multivocal en Arqueología, Antropología y Ambiente), financiada por el área de Ciencia y Sociedad del programa CYTED para el período 2013-2016, pretende contribuir a la generación de una respuesta a los retos que se le plantean a la arqueología, la antropología y a otras disciplinas, entre ellas las ambientales, que tienen al patrimonio como eje y campo transdisciplinario de acción e investigación. La red se concibe como un espacio común para cotejar, intercambiar y ampliar experiencias, una red orientada tanto a la teoría, reflexionando sobre las condiciones de producción y emergencia de los procesos de patrimonialización y su dialéctica como fenómeno social, como a la práctica, fomentando las comunidades de aprendizaje-enseñanza y la generación de valores y actitudes positivas y de transformación en torno al patrimonio, tanto natural como cultural. 
TRAMA3 es un foro que aspira a la superación del paradigma académico tradicional (cerrado, especializado y jerárquico), en favor de un modo abierto, público, transdisciplinario, participativo y generador de valores sociales, culturales y económicos avanzados dentro de la sostenibilidad, contribuyendo a la actualización de la arqueología y la antropología y a su inmersión en las nuevas dinámicas culturales, científicas, educativas y a su relación con las políticas públicas (BARREIRO Y GIANOTTI, 2014).

TRAMA3 se plantea seis objetivos específicos en torno a los que articular la reflexión y el trabajo en relación con los argumentos expuestos:

- Analizar las relaciones entre la gestión del patrimonio y los paisajes culturales (en tanto materialización en el territorio de la memoria y de las identidades colectivas) y la modernización materializada en procesos de desarrollo y transformación del territorio, desde una perspectiva dialéctica y entendiendo que el patrimonio es un campo de conflicto y de entrecruzamiento de intereses contrapuestos.

- Analizar las relaciones entre el acervo, los saberes y las prácticas de las comunidades asociados al patrimonio y a los paisajes culturales, y los procesos de hegemonía derivados de la modernización y la globalización, entendiendo el vínculo patrimonial entre las comunidades y su patrimonio como parte indisociable de las identidades de resistencia que están, a su vez, en continua transformación.

- Contribuir a la revalorización social, cultural y económica del patrimonio y del conocimiento generado a partir de él y en torno a él, mediante su construcción en modo abierto, público y participativo, dentro del paradigma de la sostenibilidad, y fomentando la multivocalidad como eje integrador.

- Contribuir a superar el modelo estándar de transferencia de conocimiento por un modelo de investigación colaborativa multiagente.

- Integrar la discusión/reflexión sobre experiencias y valores asociados al patrimonio dentro del ámbito educativo, formativo y de gestión, fomentando y facilitando la colaboración y enseñanza-aprendizaje entre investigadores, comunidad y gestores del patrimonio en el terreno de la educación formal, no formal e informal.

- Desarrollar metodologías y criterios para el trabajo arqueológico y antropológico en comunidad y con la gente.

En este contexto, los diferentes socios que integran la red TRAMA3 aportan una diversidad de experiencias que enriquece la visión y análisis del mismo. La red está formada por ocho socios 
científicos de diferentes países, así como por una comunidad de usuarios. La mayoría de los socios centran su contribución en torno a un espacio concreto en el que se desarrolla su actividad científica. A estos espacios los denominamos sitios laboratorio.

La red está coordinada desde el Laboratorio de Arqueología del Paisaje y Patrimonio (LAPPU) alojado en la Facultad de Humanidades y Ciencias de la Educación con una unidad asociada en el Centro Universitario de la Región Este (Universidad de la República), de Uruguay. El sitio laboratorio del LAPPU es el Área Protegida de la Laguna de Rocha, que forma parte de un sistema lacustre costero donde, tanto el LAPPU como otras entidade vienen dando, desde hace algunos años, procesos de incorporación y participación en la gestión del territorio de las diferentes comunidades que lo habitan. La comunidade de pescadores participa en la red a través de APALCO (Asociación de Pescadores Artesanales de las Lagunas Costeras). La experiencia en la Laguna de Rocha aporta a la red una visión integrada y holística de los procesos de apropiación y gestión de un espacio que aúna componentes materiales e inmateriales, culturales y ambientales, y lo hace tanto desde la perspectiva del análisis científico y la gestión planificada como desde la vivencia del entorno como sustento económico, territorio político y espacio simbólico y emocional. Es además, un campo fecundo para analizar la incidencia real de la participación y la multivocalidad en la toma de decisiones, la conservación y la planificación del territorio.

La heterogeneidad en los discursos de legitimación y en las formas de apropiación del patrimonio se manifiestan en los pueblos rankülches de La Pampa, en dos provincias del centro argentino (La Pampa y San Luis), que es el sitio laboratorio aportado por el programa INCUAPA (Investigaciones arqueológicas y paleontológicas del cuaternario pampeano), desarrollado desde hace más de veinte años por la Facultad de Ciencias Sociales de la Universidad Nacional del Centro de la provincia de Buenos Aires. En los últimos años se han llevado a cabo, en el marco de este programa, diferentes líneas de investigación desde una perspectiva crítica y reflexiva sobre las propias condiciones de producción del discurso académico en relación con las epistemologías alternativas que se activan en los procesos de patrimonialización.

Participa igualmente en la red el Departamento de Antropología de la Facultad de Ciencias Sociales de la Universidad de Chile. El sitio laboratorio del equipo chileno se encuentra en los valles de Rapel y Hurtado, en la cuenca alta del río Limarí (IV Región). En concreto, las actividades arqueológicas y antropológicas allí desarrolladas, en colaboración con las comunidades campesinas, revelan diferentes estrategias de resistencia y/o adaptación al proyecto de la modernidad, encarnado por las transformaciones experimentadas en el sector primario (agricultura y minería), tanto históricas como recientes. Al igual que en el caso argentino, estas estrategias de resistencia y 
adaptativas tienen una correlación directa con la forma en que el patrimonio es apropiado, reivindicado y construido por las diferentes comunidades.

Vinculados a proyectos de modernización territorial están los casos de estudio de ERA Arqueologia, la empresa de arqueología más importante de Portugal, cuyo investigador responsable para la red es António Valera. Estos casos de estudio se centran en un sitio laboratorio (Complexo Arqueológico dos Perdigões, Évora), descubierto en la década de los '80 pero que reveló todo su potencial y valor arqueológico a partir de su afección por parte de las explotaciones vitivinícolas de Reguengos de Monsaraz, en el Alentejo. Además, ERA aporta a la red su experiencia en la realización de distintas actividades de corrección de impacto a través de la difusión en el marco del proyecto de arqueología preventiva ocasionado por el gran embalse de Alqueva y sus redes de distribución eléctrica.

Por su parte, las contribuciones del Laboratório de Arqueologia Pública Paulo Duarte (LAP) de la Universidad Estatal de Campinas (São Paulo), que es un referente americano y mundial en investigación multivocal orientada al patrimonio, resulta sumamente valiosa. Los aportes de esta institución, dirigida por Paulo Funari y cuya investigadora responsable para TRAMA3 es Aline Carvalho, emanan no sólo del análisis de las condiciones sociales de emergencia de las distintas voces y legitimidades en torno al patrimonio, sino también de la investigación y acción participativas en los propios procesos de patrimonialización.

Esta relación entre patrimonio y acceso de la ciudadanía a la educación y el desarrollo integral y sostenible es especialmente relevante en el caso de la Zona Arqueológica de Caral (Perú), un lugar en el que, desde hace más de veinte años, la investigadora responsable del proyecto y para la red TRAMA3, Ruth Shady, y su equipo, han logrado convertir su rico legado arqueológico (que fue declarado Patrimonio Mundial en 2009) en el centro de la actividad científica de la región, además de en un recurso de primer orden para el desarrollo económico y cultural de la misma. Por lo tanto, Caral es un ejemplo trascendental para comprender las interacciones entre la conservación de un patrimonio excepcional y el entorno social, enfatizando la necesidad de fomentar el aprecio y la apropiación social de dicho patrimonio por parte de las comunidades locales como una estrategia que aúna las demandas de protección del patrimonio con el uso del mismo como medio para la socialización y el desarrollo.

En el año 2015, se suma formalmente a la red, el grupo de trabajo de la Secretaria técnica de Patrimonio Rapa Nui (Chile), con el sitio laboratorio de Rapa Nui; en la Isla de Pascua. 
Por último, la participación del Instituto de Ciencias del Patrimonio, perteneciente al CSIC, supone la aportación española a la red TRAMA3, cuyo investigador responsable es David Barreiro. Dentro de la variedad de proyectos que se llevan a cabo desde esta institución, el sitio laboratorio seleccionado es Altamira (Cantabria). Al igual que en el caso anterior, un sitio que fue declarado Patrimonio Mundial (en 1984, aunque en 2004 se amplió al conjunto del arte rupestre francocantábrico). Esta elección vino dada por la participación del Incipit en el Programa de Investigación para la Conservación Preventiva y Régimen de Acceso de la Cueva de Altamira (finalizado en agosto de 2014), promovido por el MECD, que incluía la realización de un estudio sobre el valor social de Altamira.

Estos sitios laboratorio como los equipos de trabajo que en ellos se encaran, desarrollan una estrategia de investigación en el que el acceso a todas esas voces y dimensiones fuerza una aproximación no sólo multidisciplinaria, sino inter y transdisciplinaria. Esta perspectiva en torno a la acción desde , para y con el patrimonio implica la pluralidad de saberes pero también de formas de aprender y de aprehender el patrimonio.

\section{ESPACIOS EDUCATIVOS INTEGRALES Y LAS REDES DE PRÁCTICA}

Cabe destacar el abordaje transdisciplinario lejos de adoptar una actitud conformista respecto de una perspectiva teórica ya aceptada (ejemplificada con los desarrollos teóricos de Pohl y Hirsch Hadorn (2008), Bammer (2013), entre otros), mantiene una postura crítica, reflexiva, atenta a los debates teóricos y, a la vez, receptiva a los cambios que se observan en la práctica de la gestión.

Como señala David Barriero (2016) "el patrimonio cultural (...) es un campo de lo social que nos desafía con sus ambigüedades y contradicciones, con sus acuerdos y sus disonancias”. En ese marco, y partiendo de la idea del patrimonio como construcción, el reconocimiento de la multiplicidad de agentes y la diversidad de valoraciones constituye el punto de partida ineludible en casi todas las experiencias descriptas, al tiempo que la apropiación social del patrimonio es señalada como el gran objetivo a alcanzar y como la finalidad última de toda acción de patrimonialización. En ese contexto, el investigador o el experto - sin dejar de lado la importancia de su aporte - pasa a ser un sujeto más en el proceso (ENDERE, 2016).

En este sentido, se puede afirmar que el objetivo de integrar la discusión/reflexión sobre experiencias y valores asociados al patrimonio dentro del ámbito educativo, formativo y de gestión, fomentando y facilitando la colaboración y enseñanza-aprendizaje entre investigadores, comunidad 
y gestores del patrimonio en el terreno de la educación formal, no formal e informal; cobra un sentido integral. Integralidad entendida como un espacio de polifonía y de permanente reflexión que reabre la discusión para la co-producción de patrimonio en un contexto educativo que conlleva varios niveles.

Bajo este objetivo es que consideramos a la red como un ámbito educativo integral desde dos niveles. Un primer nivel vinculado a las sinergias generadas entre los socios y los equipos de trabajo, donde se construye dialógicamente una red de práctica entendida como aquella que consolida saberes y conocimientos de diversos tipos, no solamente académicos (BROWN Y DUGUID 2000; BAMMER 2013). En este nivel, los integrantes directos de la red co-construyen conocimiento desde prácticas integrales (AROCENA, 2008) y multivocales. En un segundo nivel de práctica educativa, TRAMA3 fundamenta su actividad a través de varias dinámicas, a fin de generar sinergias que revaloricen el trabajo de toda la red. Tanto su principal actividad, los seminarios de trabajo, de periodicidad anual, como la realización de dos tipos de productos específicos (los protocolos y las aplicaciones) responden al objetivo sustantivo de co-crear dispositivos educativos para y con el patrimonio.

\section{Primer nivel de la práctica educativa}

No menos significativo ha sido el énfasis puesto por los autores en la necesidad de un abordaje interdisciplinario y transdisciplinario del patrimonio y la importancia de considerar el conocimiento "no científico" o "no generado en el ámbito científico" y las percepciones comunitarias, incluyendo la dimensión emocional que el patrimonio tiene para las comunidades, los grupos y aún los individuos.

Si bien ha habido un largo derrotero de literatura científica dedicada a reflexionar sobre diversos ángulos de la especificidad del fenómeno de la interdisciplina y la transdisciplina (DARBELLAY, 2015; CAETANO, 2015), cabe preguntarse si ello ha derivado en una fundamentación más sustantiva de este tipo de prácticas científicas en el ámbito patrimonial. Lo que parece suceder es que resta aún una reflexión que integre el contexto de producción del conocimiento transdisciplinario con las características propias de nuestros países y contextos. Vale un breve relevamiento para confirmar que el desarrollo de las temáticas y reflexiones en torno a la interdisciplina es diverso en el continente latinoamericano y muy incipiente aun el desarrollo en torno a un abordaje transdisciplinario (VIENNI, 2014b, 2016a). ¿Con qué objetivo sustancial se persigue una práctica científica más interdisciplinaria y transdisciplinaria? Justamente con aquel 
que aquí se expone, buscando que la actividad educativa pueda formar parte integral de la conformación cultural e identitaria de las comunidades y usuarios que reúne la red TRAMA3.

Se puede partir de varias definiciones amplias y más restringidas de interdisciplina. Lo cierto es que no todo es interdisciplina ni la interdisciplina sirve para todo. Claro que en el juego de palabras hay cierta verdad: la interdisciplina requiere de rigor científico (CAETANO, 2015) y que no todos los problemas requieren de un abordaje interdisciplinario (BAMMER, 2005).

En una publicación de enero de 2015, Chou y Ravinet argumentan la relevancia del concepo de "regionalismo" que definen como "a political project of region creation involving at least some state authority (national, supranational) who in turn designates and delineates the world's geographical region to which such activities extend, in the higher education policy sector (CHOU, RAVINET, 2015, p. 368).

Entre los ejemplos que ilustran este concepto mencionan al proceso de Bolonia en Europa o al MERCOSUR Educativo en América Latina. La definición fue diseñada luego de un estudio de lo publicado sobre regionalismo en la educación superior producto de la ciencia política y de los estudios sobre educación. De estos últimos, los autores señalan el impacto en la recomposición de espacio, escala, y poder que estos arreglos han tenido en el estado de la educación superior en el pasado, en el presente y en el futuro.

Teniendo esta definición en mente, parece posible hacernos la pregunta ¿El patrimonio como campo de saberes transdisciplinario puede constituir un regionalismo emergente? Precisamente la red TRAMA3 puede dar cuenta de un ejemplo de ello donde el proceso educativo transversaliza las prácticas de usuarios, comunidades y científicos.

La interdisciplinariedad del patrimonio se inserta en la compleja cuestión de la comunicación del conocimiento científico. Proponemos repensar las disciplinas en tanto culturas como un medio para comprender la interacción entre disciplinas que está influenciada por las condiciones sociales y por el conocimiento como construcción social (VIENNI, 2014a, 2016b).

Una cuestión recurrente es el énfasis en la necesidad de no dejar de lado las distintas dimensiones de la subjetividad, es decir, las múltiples maneras de percibir, apropiarse y usar el patrimonio, aún entre individuos de una misma cultura. Ello nos obliga a renunciar a cualquier intento de adoptar visiones simplistas o reduccionistas respecto de sus posibles posicionamientos frente a una situación particular. Por el contrario, adoptar una actitud multivocal es mucho más que escuchar, implica ir al encuentro, generar empatía, construir un diálogo, ponerse en el lugar del otro y llevarlo a la acción de manera conjunta (ENDERE, 2016). 


\section{Segundo nivel de la práctica educativa}

TRAMA3 fundamenta su actividad a través de varias dinámicas, a fin de generar sinergias que revaloricen el trabajo de toda la red.

La principal actividad dialógica son los seminarios de trabajo, de periodicidad anual, en los que se produce el intercambio entre los equipos participantes. Estos seminarios, de los que se han celebrado tres - en Montevideo, Santiago de Compostela y la ciudad de Campinas (Brasil) - son de contenido variado.

Visibilizar, en ese contexto, los procesos de patrimonialización desde una perspectiva multivocal y traducirlos en una práctica colaborativa y participativa, es aún un desafío mayor.

Lo interesante de estos ejemplos es que transcienden el debate teórico y lo llevan a la práctica, elaborando y poniendo a punto metodologías para interactuar con las comunidades (BARREIRO Y GIANOTTI, 2014). Pero lo más significativo es que ese aprendizaje no se limita a un determinado proyecto de investigación sino que sus resultados logran ser incorporados en la gestión misma de los sitios. De este modo, los propios investigadores -y en algunos casos los gestores- se convierten en interlocutores y mediadores, no sólo con las poblaciones locales sino con las diferentes áreas y niveles de gobierno, y ese intercambio impacta en cuestiones que exceden lo meramente patrimonial y se insertan en la toma de decisiones respecto del ordenamiento territorial y el uso y preservación de los recursos naturales. Asociados a estos seminarios de trabajo la red pretende celebrar jornadas o seminarios abiertos con una orientación temática, como una actividad ejecutada por algunos de los participantes de TRAMA3 y orientada a profesionales de instituciones públicas y privadas.

La red TRAMA3, respondiendo a las características de este tipo de convocatorias contempla también la realización de dos tipos de productos específicos:

a) Los Protocolos de Prácticas Recomendadas en Construcción Participativa del Patrimonio. Con esta actividad, integrada por ocho volúmenes diferentes a cargo de cada uno de los socios, se pretenden aportar pautas para diseñar y llevar a cabo prácticas patrimoniales recomendadas en base a las experiencias de los casos de estudio y trabajos de la red, desde una perspectiva participativa, multivocal y transdisciplinar.

b) Las aplicaciones patrimoniales para dispositivos móviles, que funcionarán como demostradores de las potencialidades de las tecnologías de la información en el ámbito del patrimonio. Una primera aplicación, denominada Ciencia de Ayer (un museo virtual abierto e interactivo), está siendo desarrollada desde este mismo año, por iniciativa del 
Incipit. El año que viene se iniciará el desarrollo, por parte del LAPPU; de una aplicación sobre la Laguna de Rocha.

\section{Los Protocolos de Prácticas Recomendadas en Construcción Participativa del Patrimonio}

Cada uno de estos 8 documentos, en formato electrónico, está orientado a gestores trabajen con el manejo, conservación, difusión y socialización del patrimonio, y funciona como una guía abierta. Se trata de un producto novedoso que busca revertir la unidireccionalidad de otros materiales orientados a la orientación de gestores del patrimonio. Por ello, se busca que el protocolo sea un material accesible, con un lenguaje claro y preciso y que incluya ejemplos de los países miembros.

Los objetivos generales de la Red que se vinculan con la elaboración de protocolos buscan contribuir a la revalorización social, cultural y económica del patrimonio, y del conocimiento generado a partir de él, y en torno a él, mediante su construcción en modo abierto, público y participativo, dentro del paradigma de la sostenibilidad, y fomentando la multivocalidad como eje integrador. Asimismo se quiere superar el modelo estándar de transferencia de conocimiento por un modelo de investigación colaborativa multiagente.

Aportar pautas para diseñar y llevar a cabo prácticas patrimoniales recomendadas en base a las experiencias de los casos de estudio y trabajos de la red, constituye otras de las finalidades de este producto que ya tiene sus tres primeros volúmenes terminados. La tabla 1 muestra un cronograma tentativo de estos protocolos junto con los países que trabajan en cada uno de ellos.

Tabla 1. Detalle de los protocolos a ser producidos en el marco de la red TRAMA3, los países responsables de cada uno y las fechas a ser publicados.

\begin{tabular}{|l|l|l|}
\hline Protocolo & Autor & Año \\
\hline 1. Construcción participativa del PA & Argentina & 2015 \\
\hline 2. Interpretación participativa del PA & Argentina & 2015 \\
\hline 3. Didáctica participativa del PA & Brasil & 2015 \\
\hline 4. Acceso participativo al PA & Perú & 2016 \\
\hline 5.Comunicación participativa del PA & Chile & 2014 \\
\hline 6. Gestión participativa del PA & España - Uruguay & 2016 \\
\hline 7. PA como recurso sostenible & Portugal (colabora España) & 2015 \\
\hline $\begin{array}{l}\text { 8. Evaluación de procesos } \\
\text { participativos en PA }\end{array}$ & Uruguay (colabora España) & 2014 \\
\hline
\end{tabular}

Los contenidos de cada volumen son discutidos entre todos los miembros de la red e incluyen contenidos tales como:

1. Construcción participativa del patrimonio: Equivale al protocolo "Relacionarse con la comunidad". En este protocolo se deben proporcionar pautas para establecer un vínculo efectivo 
con las comunidades en la que se desarrollan proyectos patrimoniales. Estas pautas van orientadas a que el vínculo sea participativo; es decir, que no consiste en cómo transmitir el patrimonio a la gente, sino en cómo podemos facilitar que la gente participe críticamente en los procesos de construcción del patrimonio.

2. Interpretación participativa del patrimonio: Equivale al protocolo "Interpretar el patrimonio". En este protocolo se deben proporcionar pautas para la investigación colaborativa en patrimonio y la elaboración participativa de discursos en torno al patrimonio. Cómo producir conocimiento, y generar narrativas a partir de él, de forma participativa.

3. Didáctica participativa del patrimonio: Equivale al protocolo "Diseñar unidades didácticas en torno al patrimonio". Amplía la idea anterior para incorporar todo tipo de prácticas relacionadas con la educación formal; esto es, didáctica del patrimonio en las aulas escolares (primaria y secundaria). Las pautas deben orientar a quienes imparten materias relacionadas con el patrimonio cultural.

4. Acceso participativo al patrimonio: Equivale a los protocolos "Diseñar guías para Museos" y "Diseñar guías para Sitios patrimoniales", fusionándolos y ampliando sus contenidos. Aporta pautas para la elaboración de materiales en relación con la didáctica del patrimonio en contextos no formales (fuera de las aulas escolares), en museos y sitios patrimoniales. Cómo realizar actividades, producir materiales y elaborar discursos para lograr un acceso participativo al patrimonio.

5. Comunicación participativa del patrimonio: Equivale a los protocolos "Relacionarse con el público" y "Diseñar un programa de cultura científica en patrimonio", ampliando sus contenidos. Este protocolo debe proporcionar pautas para diseñar estrategias participativas para la comunicación de contenidos y eventos relacionados con el patrimonio, a través de diversos canales de comunicación (escritos, audiovisuales, digitales).

6. Gestión participativa del patrimonio: Subsume parcialmente el protocolo "Identificar, gestionar y conservar objetos patrimoniales en su interrelación con lo ambiental”, y lo amplía. Proporciona pautas para diseñar y aplicar políticas patrimoniales participativas en patrimonio.

7. Patrimonio como recurso sostenible: Este protocolo es novedad respecto a la lista existente. Se trata de proporcionar pautas para un uso del patrimonio que promueva la actividad económica de forma sostenible, democrática, creativa y transformadora. 
8. Evaluación de procesos participativos en patrimonio: Este último protocolo, que también es novedad, pretende proporcionar criterios y pautas para evaluar cualquier actividad relacionada con el patrimonio desde una perspectiva multivocal y participativa.

En términos generales, cada protocolo funciona individualmente pero compartiendo la identidad como serie o colección. Cada protocolo está a cargo de un miembro de la red, quien cuenta con el apoyo de un segundo miembro a modo de socio - soporte (Tabla 1).

\section{LAS APLICACIONES PATRIMONIALES}

Pretendemos ser un nodo básico en esta red no sólo desarrollando una página web específica, sino invirtiendo el sentido en el que está viajando la información actualmente; proponemos que la ciudadanía participe en la totalidad de decisiones sobre este aspecto de la ciencia, desde la aplicación de técnicas novedosas hasta la decisión de qué elemento se debe "patrimonializar" haciendo saber a asociaciones, centros de enseñanza y ciudadanos en general de las posibilidades que la tecnología ofrece en este campo y cómo pueden desarrollarlas. Además incardinaremos las iniciativas que puedan surgir formando con ellas un repositorio de bienes patrimoniales accesible públicamente (INSTITUTO DE CIENCIAS DEL PATRIMONIO, 2015).

El proyecto de desarrollo de aplicaciones en el marco de la Red TRAMA3 genera plataformas virtuales con el objetivo de que los ciudadanos puedan participar activamente en el proceso de producción de conocimiento arqueológico. Para ello se implemen aplicaciones móviles que faciliten el máximo alcance del proyecto; y permitan participar a diferentes actores en la identificación de entidades patrimoniales de diferente tipo. Toda esta información podrá ser compartida por los usuarios a través de las redes sociales. La primera aplicación, llamada "Bien de acá”, se encuentra en marcha y aguarda ser lanzada oficialmente por la red a fines de 2016. Esta aplicación toma como punto de partida al sitio laboratorio de la Laguna de Rocha, en Uruguay, pero se planea incorporar otros casos de estudio conforme se avance en el trabajo de la red.

Proponemos contribuir a cerrar la brecha existente entre ciudadanos y ciencia, apoyándonos en las nuevas tecnologías. Para ello desde el Incipit se aportarán una serie de conceptos, técnicas y conocimiento experto en patrimonio que permitan a los ciudadanos conocer y aplicar técnicas científicas en este campo.

Además se ofrecen vías que capacitan a los ciudadanos para generar nuevos contenidos que a su vez nutran este tipo de plataformas. Esta propuesta busca una participación activa de los 
ciudadanos para que puedan aportar su visión del patrimonio y reclamar un papel activo en el proceso de producción de conocimiento científico.

El patrimonio cultural depende directamente de la participación ciudadana; su propia existencia y desarrollo depende de que la ciudadanía lo identifique y lo valore como tal. Proponemos la puesta en funcionamiento de lo que podemos definir como un recurso expositivo y didáctico público basado en el proceso científico de identificación, documentación, análisis y clasificación de diversas entidades patrimoniales, empleando las últimas tecnologías para la virtualización de elementos arqueológicos y su divulgación entre público no especializado. Esto se traducirá en el desarrollo de una aplicación multiplataforma (para web y dispositivos móviles) que permita mostrar información de calidad sobre entidades patrimoniales (INSTITUTO DE CIENCIAS DEL PATRIMONIO, 2015).

Pretendemos acercar al ciudadano el proceso científico que permite documentar con nuevas tecnologías, así como caracterizar, clasificar e interpretar estas entidades patrimoniales, favoreciendo la participación ciudadana a través de actividades y recursos concretos que posibiliten que cada ciudadano individualmente pueda aportar información, clasificarla e interpretarla, creando recurso divulgativo construido mediante la colaboración ciudadana. Para ello, se partirá de una colección de elementos patrimoniales básicos que representen la variedad de elementos arqueológicos que tenemos a nuestra disposición. Esta colección básica servirá de ejemplo para que con el tiempo se vaya acrecentando por medio de iniciativas colaborativas, co-financiación de nuevas incorporaciones por medio del mecenazgo o micro-mecenazgo, aportaciones ciudadanas, etc.

Este sistema ha tenido mucho éxito en los experimentos pioneros llevados a cabo por grandes marcas publicitarias, y se pretende extrapolar dicho éxito al ámbito de la divulgación de la cultura y el Patrimonio. La posibilidad de difundir nuevas entidades patrimoniales por medio de estas tarjetas tanto por medios clásicos (impresas a cualquier tamaño, desde cromos para niños a posters) como por los medios más modernos (por correo electrónico, redes sociales, etc.) otorga al proyecto el potencial necesario para tener un gran impacto en los territorios donde la red desarrolla sus actividades (INSTITUTO DE CIENCIAS DEL PATRIMONIO, 2015).

\section{RESULTADOS Y REFLEXIONES FINALES}

Como resultados de este análisis, podemos concluir que el funcionamiento de TRAMA3 implica una experiencia formativa en prácticas colaborativas, en el diálogo intersubjetivo y en 
procesos de enseñanza - aprendizaje entre los socios, que se contemplan como resultados vivenciales y experiencias capacitadoras. Asimismo, y atendiendo al segundo nivel educativo, los protocolos y aplicaciones son herramientas de gestión de demandas concretas con gran potencial de aplicabilidad en el ámbito de la gestión patrimonial y ambiental, que están vinculadas a políticas públicas relacionadas con la planificación territorial, el turismo y la educación.

Estas acciones tienen como resultado concreto que el coordinador del área de Ciencia y Sociedad en CYTED; Sandro Mendonça; ha propuesto a TRAMA3 como modelo de red exitosa del área para esta anualidad (GIANOTTI; comunicación personal).

De esta forma, una experiencia concreta de conformación de una red se transforma en un aprendizaje extrapolable a otras instancias de educación patrimonial donde la transformación de sinergias se puede dar en más de un nivel de trabajo. En este sentido, cabe mencionar que entre los beneficiarios directos de las actividades de la red se incluyen los propios socios, el público perteneciente a los contextos académicos e institucionales de los socios, las comunidades de usuarios de la red y las propias comunidades locales. Además, a través de las redes sociales o de la serie de protocolos, los resultados tendrán un círculo extendido de beneficiarios potenciales: estudiantes universitarios, agentes locales, técnicos y gestores patrimoniales, técnicos de instituciones públicas allegados a la gestión patrimonial y ambiental y comunidades de usuarios cercanas y a priori no contempladas en la estructura de la red (BARREIRO Y GIANOTTI, 2014).

Por otro lado, se pretende contribuir a una mejor capacitación de los profesionales del patrimonial, en consonancia con el espíritu que sustenta la red (multivocalidad e interdisciplinariedad). El funcionamiento de TRAMA3 deberá suponer una experiencia formativa y un transitar en prácticas colaborativas, en el diálogo intersubjetivo y en procesos de enseñanza aprendizaje entre los socios, que se contemplan como resultados vivenciales y experiencias capacitadoras. En este ámbito se distinguen:

1. La capacitación teórica y práctica derivada de la colaboración y la orientación de TRAMA3 hacia el intercambio fructífero de experiencias particulares de los socios.

2. Las acciones, asociadas a los seminarios anuales, orientadas a profesionales (investigadores, profesores de universidad, técnicos de empresas e instituciones públicas, asociaciones, etc.) de instituciones públicas y privadas.

Por último, el trabajo de la red pretende aportar mejoras en la valorización y gestión de los sitios laboratorio. El trabajo cooperativo y los avances en la teoría y práctica de la revalorización social, cultural y económica del patrimonio, tendrá un primer beneficio, directo e inmediato, en los 
procedimientos y metodologías empleados por los equipos participantes en los diferentes sitios laboratorio. Por otra parte, los Protocolos serán herramientas de gestión de demandas concretas con gran potencial de aplicabilidad en el ámbito de la gestión patrimonial y ambiental, que están vinculadas a políticas públicas relacionadas con la planificación territorial, el turismo y la educación (BARREIRO Y GIANOTTI, 2014).

\section{REFERÊNCIAS}

AROCENA; R. Presentación. Espacio Interdisciplinario, Jornada de Presentación. Editado por el equipo de Rectorado de la Universidad de la República. Montevideo: Facultad de Ciencias Sociales, Universidad de la República, Montevideo, 2008. Disp.: 〈www.ei.ur.edu.uy>.

BAMMER, G. Integration and Implementation Sciences: Building a New Specialization. Ecology and Society, vol. 10, n. 2, p. 24, 2005.

BAMMER, G. Disciplining Interdisciplinarity. Canberra: ANU Press, 2013.

BARREIRO, D. Capítulo 1. La producción de patrimonio cultural. EN: GIANOTTI; C. et al. (Coords.). Patrimonio y multivocalidad. Teoría, práctica y experiencias en torno al al construcción del conocimiento en patrimonio.Montevideo: Biblioteca Plural de la Universidad de la República, 2016. p. 17-28.

BARREIRO, D.; GIANOTTI, C. Multivocality action and heritage sciences: the TRAMA3 network. In: Actas del Congreso sobre Socialización del Patrimonio. CD ROM, 2014.

BROWN, J.; DUGUID P. The social life of information. Harvard: Harvard Business School Press, 2000.

CAETANO, G. Algunas notas generales a propósito de cómo definir la interdisciplina. In: VIENNI; B. et al. (Coord.). Encuentros sobre interdisciplina. Montevideo: Espacio Interdisciplinario y Editorial Trilce, 2015. p.153-160.

CHOU, M.-H.; RAVINET, P. The rise of "Higher Education Regionalism. An Agenda for Higher Education Research. In: J. HUISMAN, H. DE BOER, D.; DILL, D.; SOUTO-OTERO, M. (Eds.). Handbook of Higher Education Policy and Governance. Houndmills: Palgrave Macmillan, 2015. p. 361-378.

DARBELLAY, F. Rethinking inter-and transdisciplinarity: Undisciplined knowledge and the emergence of a new thought style. Futures, n. 65, p. 163-174, 2015.

ENDERE; M. L. Introducción. In: GIANOTTI, C. et al. (Coords.). Patrimonio y multivocalidad. Teoría, práctica y experiencias en torno al al construcción del conocimiento en patrimonio. Montevideo: Biblioteca Plural de la Universidad de la República, 2016, pp. 11 - 16.

INSTITUTO DE CIENCIAS DEL PATRIMONIO. Memoria Técnica. Convocatoria de ayudas del Programa de Cultura Científica y de la Innovación. Patrimonio Aumentado 3D. Tecnologías interactivas para la participación ciudadana en la Ciencia. FCT-13-6882. Manuscrito; 2015.

POHL, C.; HIRSCH HADORN, G. Principles for designing transdisciplinary research. Munich: Swiss Academies of Arts and Sciences, Oekom Verlag, 2008.

VIENNI, B. Interdisciplinary Socialization of Archaeological Heritage in Uruguay. Journal of Cultural Heritage Management and Sustainable Development, vol. 4 n. 1, p. 95-106, 2014a.

VIENNI, B. La socialización del patrimonio arqueólogico como problema interdisciplinario. Una propuesta para Uruguay. Revista PH Investigación Disp.: http://www.iaph.es/phinvestigacion/index.php/phinvestigacion. Acesso: marzo de 2014, 2014b.

VIENNI, B. Los estudios sobre interdisciplina: construcción de un ámbito en el campo CTS. Quilmes: Revista Redes de la Universidad Nacional de Quilmes, 2016a.

VIENNI, B. Capítulo 2. El rol de la interdisciplina en el ámbito del patrimonio arqueológico uruguayo y su socialización. In: GIANOTTI, C. et al. (Coords.). Patrimonio y multivocalidad. Teoría, práctica y experiencias en torno al al construcción del conocimiento en patrimonio.Montevideo. Biblioteca Plural de la Universidad de la República, 2016b. p. 29-40. 
Agradecimientos

Los autores desean agradecer especialmente al Programa Iberoamericano deCiencia y Tecnología para el Desarrollo (Cyted), al Centro Universitario Regional Este Sede Rocha (Universidad de la República) y al Espacio Interdisciplinario (Universidad de la República).

\section{RESUMEN}

La red TRAMA3 (Trabajo en Red para la Acción Multivocal en Arqueología, Antropología y Ambiente), financiada por el área de Ciencia y Sociedad del programa CYTED para el período 2013-2016, busca contribuir a la generación de una respuesta a los retos que se le plantean a la arqueología, la antropología, y a otras disciplinas ambientales que tienen al patrimonio como eje y campo transdisciplinario de acción e investigación. Esta red constituye un ámbito educativo integral desde dos niveles. Un primer nivel vinculado a las sinergias generadas entre los socios y los equipos de trabajo. En un segundo nivel, TRAMA3 fundamenta su actividad a través de varias dinámicas, a fin de generar sinergias que revaloricen el trabajo de toda la red.

Palabras clave: Educación. Integralidad. Patrimonio.

\section{LAS PRACTICE NETS AS EDUCATIONAL ESPACIOS INTEGRALES: LA RED TRAMA3}

\section{ABSTRACT}

The TRAMA3 network (Networking for Multivocal Action in Archaeology, Anthropology and Environment), funded by the Science and Society area - CYTED program - for the period 2013-2016; seeks to contribute to the generation of a response to the challenges posed to archeology, anthropology, and other disciplines that have environmental heritage as an axis and transdisciplinary field of action and research. This network is an integral educational field from two levels. A first level is linked to the synergies between partners and work teams. On a second level, TRAMA3 bases its activity through various dynamics, to generate synergies that revalue the work of the entire network.

Keywords: Education. Integrity. Heritage.

\section{LAS REDES DE PRÁTICA COMO ESPAÇOS EDUCATIVOS INTEGRAIS: A REDE TRAMA3}

\section{RESUMO}

A rede TRAMA3 (Rede de Ação Multivocal Arqueologia, Antropologia e Meio Ambiente), financiado pelo programa CYTED área de Ciência e Sociedade para o período 2013-2016, visa contribuir para a geração de uma resposta aos desafios para a arqueologia, antropologia e outras disciplinas que assumem o patrimônio ambiental como um eixo e como um campo transdisciplinar de ação e pesquisa. Esta rede é um domínio da educação constituído por dois níveis. Um primeiro nível, ligado às sinergias entre parceiros e equipes de trabalho. No segundo nível, TRAMA3 baseia a sua atividade na geração de sinergias que revalorizar o trabalho de toda a rede.

Palavras-chave: Educação. Integralidade. Patrimônio.

Submetido em: Ago. 2016

Aprovado em: Dez. 2016 